\title{
APPLICATION OF MCDM METHODS FOR A GROUP OF NONHOLONOMIC MOBILE ROBOTS TO DETERMINE THE BEST ROUTE AND THE MOST SUITABLE ROBOT TO THE GIVEN TASK
}

\author{
Alpaslan Yufka \\ Department of Electrical and Electronics Engineering \\ Anadolu University \\ Eskişehir, TURKEY \\ E-mail: ayufka@gmail.com \\ Müjgan Sağır Özdemir \\ Department of Industrial Engineering \\ Eskişehir Osmangazi University \\ Eskişehir, TURKEY \\ E-mail: msagir@ogu.edu.tr
}

\begin{abstract}
In this study, a fire-fighting scenario in an office environment wherein three different nonholonomic differential-drive mobile robots are used is considered as a case study. The 2D configuration space of the office environment is divided into grid cells by using the method of "Occupancy Grid Map" such that each grid cell is associated with each interrelated node. Each robot constructs a reachability three by using these nodes and Breadth-First Search (BFS) algorithm. The back-tracking algorithm is used to obtain the finite solution set of paths from the motion planning. The set of alternatives is constructed by randomly selecting routes from the finite solution set of paths. Each robot determines its own best route by applying Multi-Criteria Decision Making (MCDM) methods such that "Elimination et Choix Traduisant la Realite (ELECTRE I)" and "Technique for Order of Preference by Similarity to Ideal Solution (TOPSIS)". Criteria for the path selection is weighted by applying the method of "Analytic Hierarchy Process (AHP)". Then, each robot except the leader robot sends its best path-info to the leader so that the leader robot determines the most suitable robot that conforms to the fire-fighting task by using AHP. To analyze the effect of criteria's weights on the alternatives and perform sensitivity-graphs, Expert Choice 11 software is used. The robot determined by the leader executes the task by tracking its own best path.
\end{abstract}

Keywords: Mobile robots, motion planning, Occupancy Grid Map, Breadth-First Search, node, back-tracking, leader, Multi-Criteria Decision Making, ELECTRE I, TOPSIS, AHP, Expert Choice, sensitivity-graphs. 


\section{Introduction}

A mobile robot (MR) constructs its route from its start point to a specific point by using motion planning techniques in order to execute the given task. In motion planning, the total length of the path is generally considered as a main criteria (Ramos, 2010) and A* algorithm is used to construct a minimal total-length path (Murphy, 2000). This criterion is not only the one, but criteria such as "changes in direction", "length in reverse gear", "index of smoothness", "average distance" are also considered (Ramos, 2010). MR is able to generate more than one path from its goal point to the specific point since the configuration space is applicable. This occurs the finite-set of alternative paths such that the best path is determined by MR according to previously defined criteria.

In (Ramos, 2010), nonholonomic car-like single MR is considered, and the best path is chosen by using the methods of ELECTRE I, II and PROMETHEE I, II. It is also that there is no info how the weights of criteria are specified. These Multi-Criteria Decision Making (MCDM) methods are generally applied in the field of economics, environmental issues, logictic, etc. and not commonly in the field of MR.

In this study, a fire-fighting scenario in an office environment wherein three different nonholonomic differential drive multi-MR are used is considered as a case study. Each MR determines its own best route by applying MCDM methods such that ELECTRE I and TOPSIS. Criteria for the path selection which are "total length", "number of rotations", "the amount of open space", and "the floor-roughness" is weighted by applying the method of "Analytic Hierarchy Process (AHP)" (Saaty, 1989). Then, each robot except the leader robot sends its best path-info to the leader so that the leader robot determines the most suitable robot that conforms to the fire-fighting task by using AHP. To analyze the effect of criteria's weights on the alternatives and perform sensitivitygraphs, Expert Choice 11 software is used. The robot determined by the leader executes the task by tracking its own best path.

\section{Literature Review}

In general, navigation is the problem of finding a collision-free motion for the robot system such a MR from a named place to another in configuration space that is known, unknown or partially known environment (Choset, 2005). In practically, MR cannot generate a direct motion path from a start (home) point to a goal (destination) point in configuration space so that path planning techniques for MRs must be used in this situation. Occupancy Grid Map (Moravec, 1985) method is used to divide 2D configuration space into grid cells. Each cell is associated with interrelated nodes by using offline planning. Breadth-First Search (BFS) algorithm (Murphy, 2000) which is based on visiting all neighbor-nodes, is used for obtaining reachability tree from the start point to the goal point. Back-tracking method is applied on this reachability tree to obtain the set of paths. 


\section{Hypotheses/Objectives}

A two-phased decision methodology is used. Required data is mostly obtained from the motion planning by using nodes and environment info. Each MR determines its own best route and the leader robot chooses the most suitable robot that conforms to the task by using AHP. Then, the robot determined by the leader executes the task by tracking its own best path.

\section{Research Design/Methodology}

The mixed method which has 2 steps is used in this study. Data is mostly obtained from the motion planning by using nodes and environment info.

1. Firstly, each robot determines its best route by using ELECTRE I and TOPSIS. The result is the same in respect of both methods. The weights of criteria is assigned by the AHP where data and author's expert view are used for pairwise comparisons. Criteria for the path selection are "total length", "number of rotations", "the amount of open space", and "the floor-roughness".

2. Secondly, the leader robot determines the most suitable robot that conforms to the task by using AHP. The weights of criteria is assigned by the AHP where data and author's expert view are used for pairwise comparisons. Criteria for the robot selection are "velocity (linear/angular)", "the capacity of the battery", "the capacity of the fire extinguisher", and "criteria for the path previously determined by each MR".

\section{Data/Model Analysis}

TOPSIS, ELECTRE and AHP methods are used for different phases of this study. Figure 1 represents one of the paired comparison matrix for criteria - changes in direction", "length in reverse gear", "index of smoothness", and "average distance" related to the path with the inconsistency index as 0.06 . Figure 2 shows that if the weight for criterion Speed increases, Robot 1 becomes the best alternative.

\begin{tabular}{|c|c|c|c|c|}
\hline & Alt Kriter 4.1 : Uzunluk & Alt Kriter 4.2: Dönüş & Alt Kriter $4.3:$ Ferahlık & Alt Kriter 4.4: Zemin Pïriizlïği \\
\hline Alt Kriter 4.1 : Uzunluk & & 3,0 & 4,0 & 6,0 \\
\hline Alt Kriter 4.2 : Dönïş & & & 3,0 & 5,0 \\
\hline Alt Kriter 4.3 : Ferahlık & & & & 3,0 \\
\hline Alt Kriter 4.4 : Zemin Pürüzlìğï & Incon: 0,06 & & & \\
\hline
\end{tabular}

Figure 1. Paired comparison matrix for path criteria

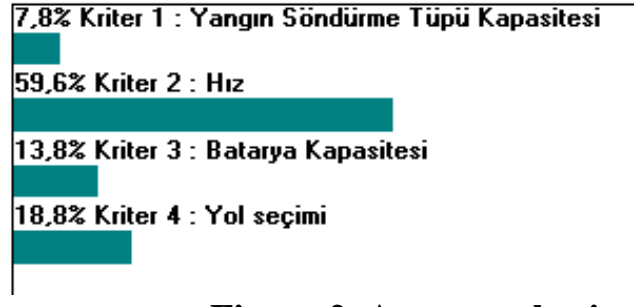

Figure 2. An example view for sensitivity analysis

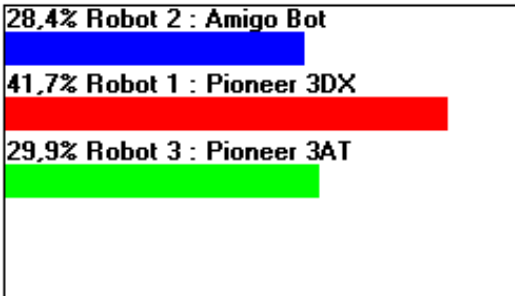


IJAHP Article: Mu, Saaty/A Style Guide for Paper Proposals To Be Submitted to the International Symposium of the Analytic Hierarchy Process 2014, Washington D.C., U.S.A.

\section{Limitations}

Assumptions are:

- Grid cells, nodes and relations of nodes are offline planned.

- Configuration space and objects are known by MR.

- The goal/destination point is reachable.

- MR has a perfect localization.

\section{Conclusions}

In this study, MCDM techniques which are ELECTRE I, TOPSIS and AHP are applied on the field of robot motion planning. We have not come across a study uses MCDM techniques to define the best route and/or the best robot in related topics. This could motivate researchers on the area of Electric/Electronic Engineering and the Computer Science for a multidisciplinary work. This study already is an example for that.

\section{Key References}

Choset, H. \& et al (2005). Principles of Robot Motion: Theory, Algorithms, and Implementations. MIT Press.

Moravec, H.P. \& Elfes, A. (1985). High resolution maps from wide angle sonar. Robotics and Automation, IEEE International Conference, (2), 116-121.

Murphy, R.R. (2000). Introduction to AI Robotics. MIT Press.

Ramos, J.M.M \& et all (2010). Application of MCDM Techniques to Maneuver Planning in Nonholonomic Robots. Expert Systems with Applications, 37, 3962-3976.

Saaty, T., \& Alexander, J. (1989). Conflict Resolution: The Analytic Hierarchy Process. New York, New York: Praeger.

\section{Appendices}

The configuration space is shown as in Figure 3. Figure 4 represents accessibility tree for Robot 1 , as an example. 
IJAHP Article: Mu, Saaty/A Style Guide for Paper Proposals To Be Submitted to the International Symposium of the Analytic Hierarchy Process 2014, Washington D.C., U.S.A.

(a)

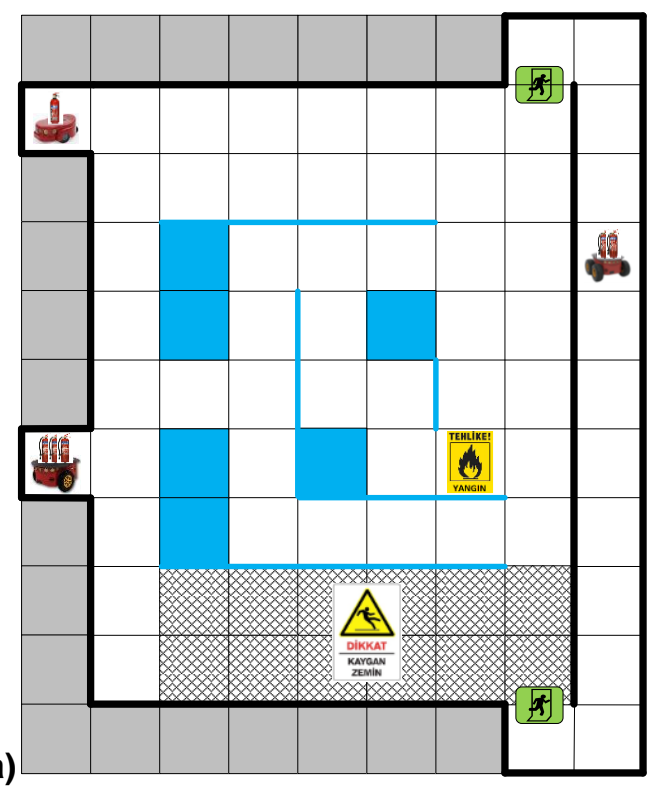

(b)

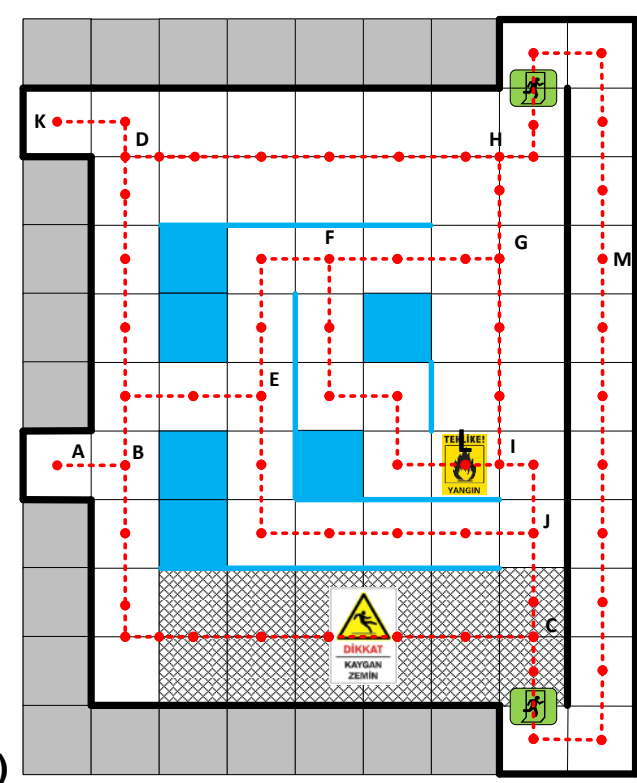

Figure 3. (a) 2D Configuration Space and (b) Offline Planning

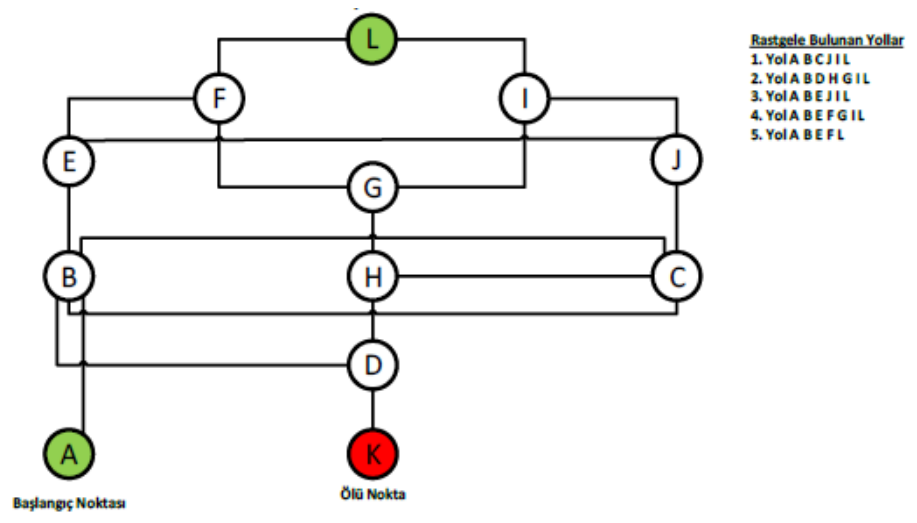

Figure 4. Accessibility tree for Robot 1 